\title{
Computational Investigation of Secondary Acoustic Radiation Force Between Cells and Particles Out of Nodal Pressure Line
}

\author{
Hamid Reza Aghaie, Mohsen Saghafian*, Ali Mohammadi and Davood Saeidi \\ Department of Mechanical Engineering, Isfahan University of Technology, Isfahan, Iran
}

*Corresponding author: Mohsen Saghafian, Department of Mechanical Engineering, Isfahan University of Technology,

Isfahan, Iran

\section{ARTICLE INFO}

Received: 慧 June 21, 2021

Published: 㓞 August 03, 2021

Citation: Hamid Reza Aghaie, Mohsen Saghafian, Ali Mohammadi, Davood Saeidi. Computational Investigation of Secondary Acoustic Radiation Force Between Cells and Particles Out of Nodal Pressure Line. Biomed J Sci \& Tech Res 37(4)-2021. BJSTR. MS.ID.006045.

Keywords: Perturbation Theory; Ultrasonic Field; Secondary Acoustic Radiation Force

\begin{abstract}
A finite element method with perturbation theory for calculating the secondary acoustic radiation force between cells and solid particles is implemented and simulated results were compared with experimental results. To do so, two sets of particles, including polystyrene particles (sized 4.8 and $25 \mu \mathrm{m}$ ), and red blood cell and silica particle (6 and $20 \mu \mathrm{m}$ ) were exposed to a 2-MHz ultrasonic field and the secondary acoustic radiation force on moving particles, located in various positions in between a pressure node and pressure antinode is obtained. In this process, we consider both compressibility and density to compute the secondary force. Due to the stiffness of a fixed particle, the magnitude of secondary acoustic radiation force is relatively different in polystyrene particles (first case) and red blood cell and silica particle (second case) specially in close proximities. We show that when the contact angel between the moving and fixed particle is nearly 0 (center to center line is perpendicular to the wave propagation direction), the secondary acoustic radiation force is dominant relative to primary force in this region. In mentioned condition, simulation and computational data shows good agreement with experimental data and these Results usefully enable better cell trapping and separating channel design.
\end{abstract}

\section{Introduction}

The wide applications of acoustofluidic devices which have been studied in recent years, play a key role in cell manipulation and separation $[1,2]$. In such cases, the first part of acoustic force, which is called primary acoustic radiation force, has been widely investigated in last few decades [3-5]. This force acts on cells and particles with different properties related to the containing fluid and causes some effects on cell deformation which have also been studied experimentally and theoretically in recent years in which, the investigators have tried to improve their simulations by using the solid models [6,7]. Recently, Aghaie, et al. [8] found that the viscoelastic model predicts the cell deformation behavior more relevant to the experimental data in comparison with previous models which were applied to cells with no respect to inter-particle force. When two or more suspended objects (particle or bubble) are subjected to an external acoustic field, the inter-particle force appears [9]. This mutual force between particles which is known as secondary acoustic radiation force results in attraction or repulsion and under this condition and each particle is exposed to both the incident field (equal to the primary force) and the scattered field from the neighboring [10]. This inter-particle force may affect some applications like cell trapping, cell clustering, and cell sorting [11]. Most of the studies have developed and reported the secondary force on bubbles in the acoustic field [12-16]. In 1906 a theoretical investigation of acoustic interaction force between a pair of air bubbles was derived by Bjerknes [9].

Crum [13] extended his analysis to the experimental study on pulsating bubbles in a sound field. By considering a wide distance range between two small gas bubbles in a viscous liquid, Doinkov [16] showed a repulsion between the bubbles which was in contradiction with Bjerknes formula. In addition to the bubbles, 
this mutual interaction force has been expressed both theoretically and experimentally for particles [17-19] and fluid spheres [20] in a plane wave field. Silva and Bruus [21] obtained a theoretical formula for interaction forces in the Rayleigh scattering limit. They calculated the inter-particle force by a potential field in an inviscid fluid. Sephrirahnama, et al. [10] proposed a numerical solution by using a weighted residue procedure to compute the total acoustic radiation forces. Their investigations were carried out in close proximity to pressure node with no restriction on the spherical particles size. Recently, Habibi, et al. [22] conducted a numerical simulation to find out the impact of particle size and material properties on the acoustic forces, including the secondary force. They demonstrated a various behavior in regimes and forces direction due to the resonant modes. The current literature lacks a research on the secondary acoustic force out of the nodal pressure. The monopole part of the secondary acoustic force vanishes in the pressure node. Most of the previous studies were also restricted to the bubbles in which, the dipole effect is eliminated, whereas the compressibility influences the secondary force.

Moreover, a few researchers have simulated the secondary acoustic force by numerical method. For example, Habibi, et al. [22] placed the particles in the wave propagation direction and considered a same direction for both the primary and the secondary forces. In this paper, we present a numerical and experimental study for two groups of particles to calculate the primary and secondary acoustic forces. In order to achieve our purpose, firstly, we select two polystyrene particles of different sizes ( 4.8 and $25 \mu \mathrm{m}$ ). In the second section we use a red blood cell and silica particle with sizes 6 and $22 \mu \mathrm{m}$, respectively. By considering a fixed position outside of the pressure node for larger particle, and a moving pathway for the smaller particle, the primary and secondary acoustic forces are calculated. As a consequence, the dependence of the secondary acoustic force on the inter-particle distance is obtained. Additionally, a summary on the experimental procedure is presented and all results are compared with the experimental analysis.

\section{Theory and Formulation}

\section{Primary Acoustic Force}

In the case where particles are suspended in a fluid and driven by an acoustic wave without considering an interaction phenomenon, they will be affected by the primary acoustic force. For a compressible particle in an ideal fluid, this force is written as [23]:

$$
F=4 \pi R^{3} E_{a v} k \phi \sin (2 K z)(1)
$$

where $R$ is the particle radius, $\mathrm{k}$ is the acoustic wave number, $E_{a v}$ is the acoustic energy density, and $z$ is the distance till the nearest pressure node. $\Phi$ denotes the acoustic contrast factor defined as:

$$
\phi=1-\frac{\beta_{p}}{\beta}+\frac{3\left(p_{p}-p\right)}{2 p_{p}+p} \text { (1-a) }
$$

Here $B$ is the compressibility, $\rho$ is the density and the subindex $p$ and $f$ correspond to particle and fluid, respectively. Positive or negative value of contrast factor determine the particle/cell moving direction to the pressure node or antinode, respectively [23].

\section{Secondary Acoustic Force}

In the acoustic field, when particles are in close proximity to each other, the inter-particle force starts becoming significant. Investigation of secondary acoustic force on particles back to the study of Weiser, et al. [19]. They derived this interaction force for two solid particles with radius of $\mathrm{R}_{\mathrm{p} 1}$ and $\mathrm{R}_{\mathrm{p} 2}$ in two expressions:

$$
\begin{aligned}
& F_{0}=\frac{2 \pi\left(\rho_{\rho}-\rho\right)^{2} V_{a c}^{2} R_{\rho 1}^{2} R_{\rho 2}^{2}}{3 \rho d^{4}} \\
& F_{s}=F_{0}\left(3 \cos ^{2}(\theta)-1\right)(2-\mathrm{a})
\end{aligned}
$$

where $V_{a c}$ is the incident field velocity, $\mathrm{d}$ is the inter-particle distance, and $\theta$ is the angle between the propagation direction of the incident wave and the centerline connecting the two particles. For the case where the particles lined up in the wave propagation direction $(\vartheta=0)$ the secondary acoustic force is repulsive. On the other hand, the force between the particles lined up perpendicular to the propagation direction $(\vartheta=90)$ is negative and attractive. Gröschl [1] by extending the Crum [13] study, carried out the formula including both the orientation and particle position effects. This force for two similar particles is given by:

$$
F_{s}(z)=4 \pi R^{6}\left\{v^{2}(z) \frac{\left(\rho_{\rho}-\rho\right)^{2}\left(3 \cos ^{2} \theta-1\right)}{6 p d^{4}}-p^{2}(Z) \frac{\rho_{f} \omega^{2}\left(\beta_{\rho}-\beta\right)^{2}}{9 d^{2}}\right\}
$$

where $\omega$ is the angular frequency. $v(z)$ and $p(z)$ are the velocity and pressure in the particle position, respectively, of the unperturbed incident acoustic field. The first term (dipole term) represents either attraction $(\vartheta=90)$ or repulsion $(\vartheta=0)$ and it vanishes at the pressure anti node. Likewise, the second term (the monopole term) depends on the compressibility representing an attractive force and it vanishes in the pressure node. For particles in between the pressure node and the velocity node, both terms should be considered. In the present study, in order to compute the primary and secondary acoustic forces, we use the perturbation method by considering a series for the pressure, velocity and density. These fields begin with the stationary part $\left(p_{0^{\prime}} v_{0^{\prime}} \rho_{0}\right)$, followed by the first order $\left(p_{1}, v_{1}, \rho_{1}\right)$ and second order terms $\left(p_{2^{\prime}} v_{2^{\prime}}\right.$ $\rho_{2}$ ). Then the pressure, velocity and density can be written as [24]:

$$
p=p_{0}+p_{1}+p_{2}(4-\mathrm{a})
$$




$$
\begin{aligned}
& v=v_{0}+v_{1}+v_{2}(4-\mathrm{b}) \\
& \rho=\rho_{0}+\rho_{1}+\rho_{2}(4-\mathrm{c})
\end{aligned}
$$

The acoustic radiation force acting on a particle, in an inviscid fluid can be calculated by integrating the time averaged second order pressure field on the surface of particle as given in the following equations [24]:

$$
\begin{aligned}
& <p_{2}>=\frac{1}{2 \rho c^{2}}<p_{1}^{2}>-\frac{1}{2} \rho<v_{1}^{2}> \\
& <F>=\int\left(\frac{\rho_{0}}{2}<\overrightarrow{v_{1}^{2}}>n \frac{1}{2 \rho c^{2}}<p_{1}^{2}>n-\rho\left(n \cdot v_{1}\right) v_{1}\right) d s
\end{aligned}
$$

Here, $\mathrm{C}$ is the speed of sound in fluid, $\mathrm{n}$ is the normal unit vector, $\mathrm{s}$ is the surface of the particle, and $<>$ is the time average operator. In Eq. (6) the first two terms are the kinetic and hydrostatic energy, and the third term is the momentum flux across the boundary surface of the particle [25]. The first order parameters $\left(\mathrm{p}_{1}, \mathrm{v}_{1}\right)$ consist of the incident and scattered parts, for instance, the pressure can be expressed as:

$$
\begin{aligned}
& p_{1}=p_{\text {in }}+p_{s c} \quad(7-\mathrm{a}) \\
& p_{1}^{2}=p_{i n}{ }^{2}+2 p_{i n} p_{s c}+p_{s c}^{2} \quad(7-\mathrm{b})
\end{aligned}
$$

where index in and $s c$ refer to the incident and scattered wave, respectively. In the small size particle cases $(\mathrm{R}<<\lambda)$ the $p_{s c}{ }^{2}$ term will be negligible compared to $p_{i n}{ }^{2}$ and $2 p_{i n} p_{s c}$ [25]. However, for large particles the scattered terms must be considered. Also, the scattered-scattered and scattered-incident terms play a key role in the secondary acoustic force calculation because the acoustic inter-particle force depends strongly on the particle distance and scattering effects. In calculating the primary acoustic force acting

Table 1: Parameters used in modeling. on a particle, Gorkov's equation cannot provide a precise solution because it does not take the scattering effects into account. Thus, due to the small distance between the particles in the present work, we employ the perturbation theory by using the first order terms to calculate the primary and secondary acoustic force.

\section{Simulation Procedure}

\section{Algorithm and Boundary Condition}

The commercial finite element software (COMSOL Multiphysics @ V.5.3) is implemented so as to simulate 2D and 3D models of two particles/cell in an acoustic field. The simulation model consists of two particles and particle/cell, experiencing acoustic force in different positions. The aim of simulation is to evaluate the primary and secondary acoustic force and the correlation between them in special positions out of nodal pressure. In the current study, we use the Acoustic Pressure physics to obtain the acoustic pressure field at the first steps. Furthermore, we employ the Solid Mechanics physics to obtain the impacts of linear elastic behavior particle as a scattering point. The computational domain is modeled by coupling the Pressure Acoustic and Solid Mechanics physics and acoustic radiation forces (primary and secondary) are evaluated by integrating from Eq.6 over the particle surface. The material and domain properties considered in the model, are given in (Table 1). The fluid characteristics are obtained from the COMSOL library. At the first case, both the fixed and moving particles are selected as polystyrene with sizes 4.8 and $25 \mu \mathrm{m}$, respectively. Secondly, we consider a red blood cell ( $6 \mu \mathrm{m}$ of diameter) as a moving particle

\begin{tabular}{|c|c|c|c|c|c|}
\hline Parameters & Value & Unit & Parameters & Value & Unit \\
\hline Fluid domain & & & Red blood cell & & \\
\hline Density, $\rho$ & 997 & Kg.m ${ }^{-3}$ & Diameter, $\mathrm{d}_{3}$ & 6 & $\mu \mathrm{m}$ \\
\hline Wavelength, $\lambda$ & 758 & $\mu \mathrm{m}$ & Density, $\rho_{\mathrm{p}}$ & 1100 & Kg.m $\mathrm{m}^{-3}$ \\
\hline Speed of sound in medium, C & 1480 & $\mathrm{~m} \cdot \mathrm{s}^{-1}$ & Speed of sound, $C_{p}$ & 1700 & $\mathrm{~m} . \mathrm{s}^{-1}$ \\
\hline Height, $\mathrm{H}$ & 380 & $\mu \mathrm{m}$ & Young's Modulus, E & 629 & pa \\
\hline Width, W & 400 & $\mu \mathrm{m}$ & Poissons's ratio, $v$ & 0.499 & - \\
\hline Frequency, f & 1.95 & $\mathrm{MHz}$ & Silica & & \\
\hline Wave number, $\mathrm{K}$ & 8278.51 & $\mathrm{~m}^{-1}$ & Diameter, $d_{4}$ & 20 & $\mu \mathrm{m}$ \\
\hline Pressure magnitude, $\mathrm{P}_{\mathrm{d}}$ & 75 & Кра & Density, $\rho_{\mathrm{p}}$ & 2330 & Kg.m ${ }^{-3}$ \\
\hline Polystyrene & & & Speed of sound, $C_{p}$ & 8500 & $\mathrm{~m} . \mathrm{s}^{-1}$ \\
\hline Fixed particle diameter, $\mathrm{d}_{1}$ & 25 & $\mu \mathrm{m}$ & Young's Modulus, E & 73 & Gpa \\
\hline Moving particle diameter, $\mathrm{d}_{2}$ & 4.8 & $\mu \mathrm{m}$ & Poissons's ratio, $v$ & 0.15 & - \\
\hline Density, $\rho_{\mathrm{p}}$ & 1070 & Kg.m ${ }^{-3}$ & & & \\
\hline Speed of sound, $C_{p}$ & 2400 & $\mathrm{~m} \cdot \mathrm{s}^{-1}$ & & & \\
\hline Young's Modulus, E & 3 & Gpa & & & \\
\hline Poissons's ratio, $v$ & 0.34 & - & & & \\
\hline
\end{tabular}
and silica particle as a fixed particle $(20 \mu \mathrm{m}$ of diameter). These sizes were chosen to compare the simulation results with experimental data. While thanks to the axisymmetric domain we use a $2 \mathrm{~d}$ model, in order to simulate the current study presented in results and discussion part, a $3 \mathrm{~d}$ model is implemented. 
The applied boundary conditions are shown in (Figure 1). It is indeed a $3 \mathrm{~d}$ case in which, surrounding fluid is deliberately represented as a rectangular geometry instead of cylindrical one to clearly show the boundaries. And particles are also depicted by a circular domain. The standing wave field is generated as $\mathrm{p}=\mathrm{pd} \cos (\mathrm{ky})$ propagating along the $\mathrm{y}$-axis. We use the plane wave radiation for boundaries to surround the model with minimum acoustic reflection. The larger particle is fixed in between the pressure node and pressure antinode and the smaller particle is placed on particular positions in the neighboring of the stationary particle. Actually, we consider a definite pathway for the moving particle and determine the acoustic force based on this positioning. The primary acoustic force is obtained by Eq. (6) along the y-axis (the wave propagation direction) and compared with Gorkov's theory. Integrating Eq. (6) over the smaller particle (moving particle) surface in the center-to-center line direction, leads to the secondary acoustic force and the results are compared with the experimental data in following sections.

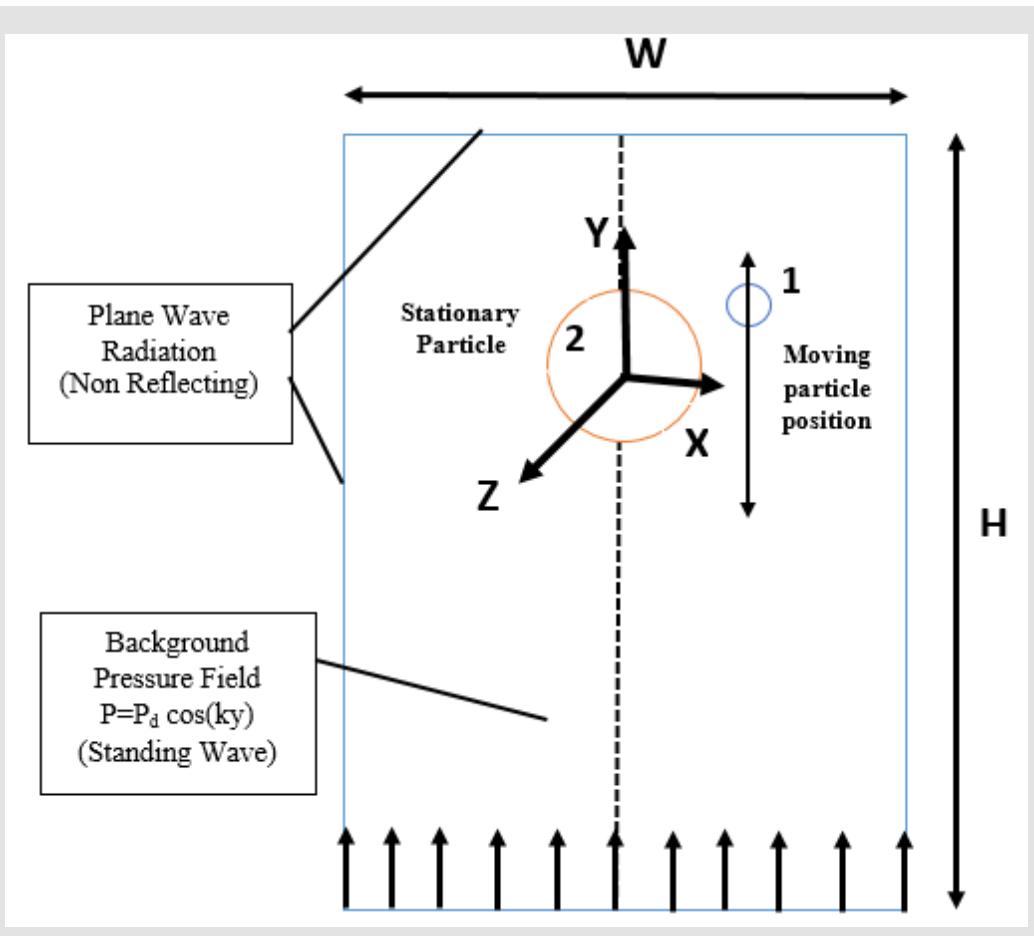

Figure 1: Geometrical configuration and boundary conditions.

\section{Grid Generation}

In order to obtain an appropriate mesh size, many different grid networks were tested. Finally, 423513 triangular elements are chosen as shown in (Figure 2a). We adopt a uniform mesh in outer region of the particles and moreover, a very refined meshes are used close to the particles. The maximum mesh size limited to $20.9 \mu \mathrm{m}$ for the fluid domain and $3.59 \mu \mathrm{m}$ for the solid domain. Also, the grow rate of the fluid and solid domain is set to 1.3. The grid independency is studied in (Figure 2b). To find an independent grid system, the primary and secondary acoustic forces are obtained for different cellular element sizes.

\section{Validation}

In order to investigate the validity of the current model, a numerical study is simulated, and its results are compared with [22]. A 2D axisymmetric model and grid network elements are demonstrated in (Figure 3). The polystyrene particle size varies from $0.03 \lambda$ up to $\lambda$ and its properties plus fluid domain parameters are mentioned in [26]. (Figure 4) illustrates the acoustic pressure contour and the primary radiation force which is compared with [22] results and Gorkov's equation. As shown, our results are compatible with their models. However, there's a difference between the numerical results and Gorkov's theory. For the larger particles, it happens due to the restriction of Gorkov's equation in considering the scattering effects. In the second case where there are two identically sized particles, the secondary acoustic force is presented and compared with [22] study. (Figure 5) illustrates the primary and total force acting on single and two particles. They determined the magnitude of secondary acoustic force by evaluating the primary and total forces. At first, they calculated the primary acoustic force for a single particle. By adding the second particle, they computed the acoustic force acting on first particle which was called total acoustic force. Since these forces are applied in the same direction, they calculated the secondary acoustic force by:

$$
F_{\text {tot }}=F_{\text {prim }}+F_{\text {sec }} F_{\text {sec }}=F_{\text {tot }}-F_{\text {prim }}
$$




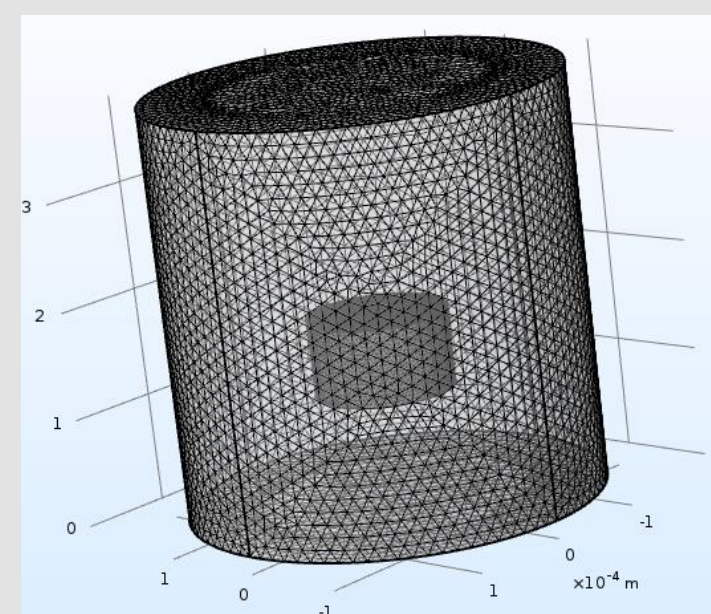

A

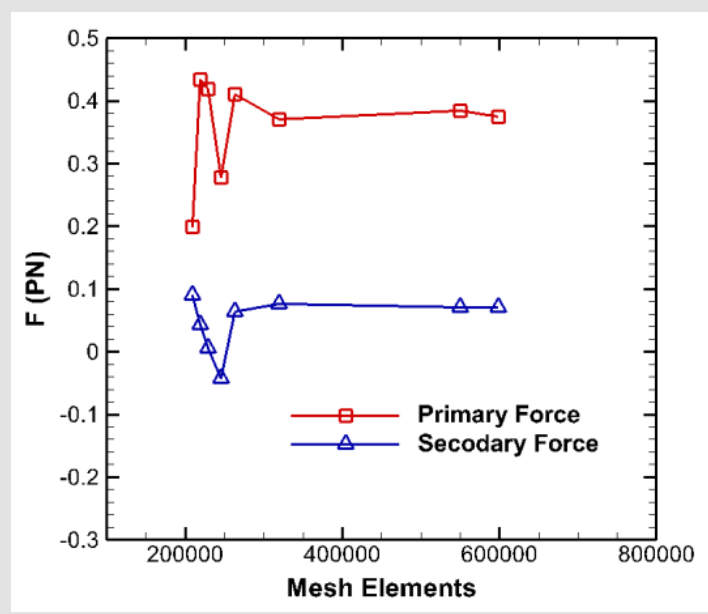

B

Figure 2: a) an uniform mesh in fluid domain and a dense mesh region around particles b) Mesh independency plot.

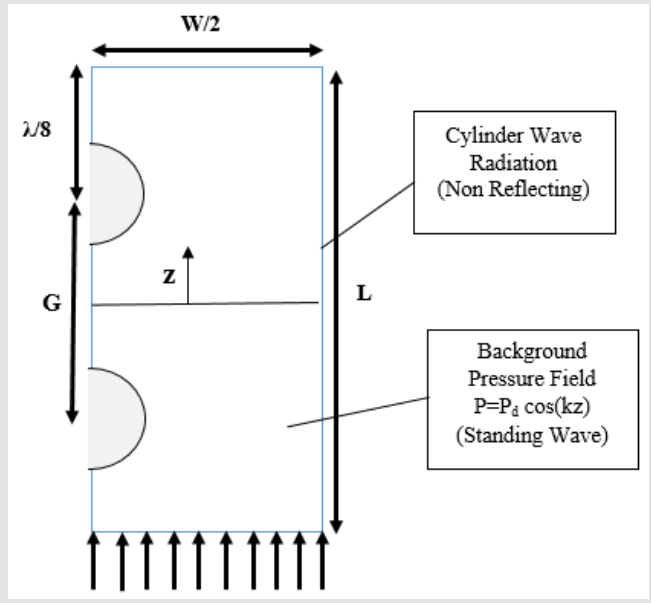

A

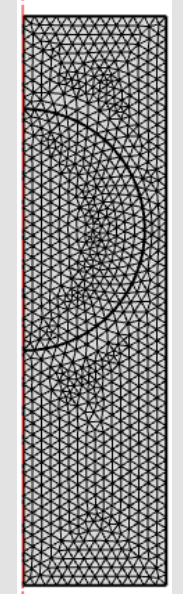

B

Figure 3: a) Schematic of axisymmetric geometry and boundary conditions; b) Mesh elements.

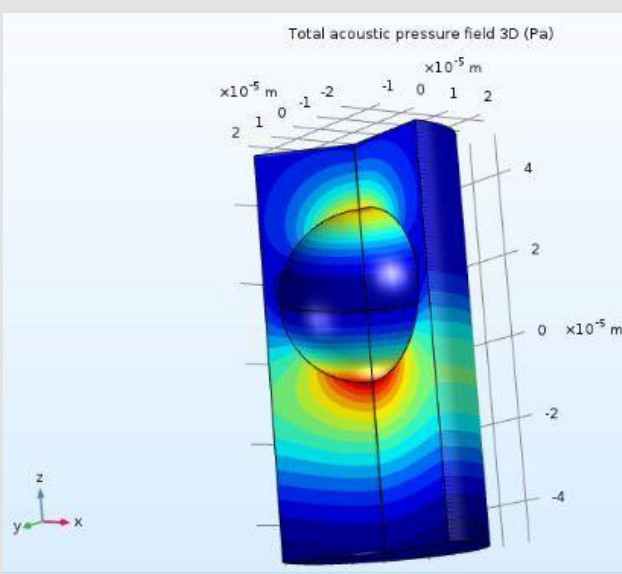

A

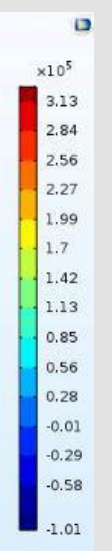

Figure 4: a) Acoustic pressure field 3D b) Primary radiation force of present study compared with results of [22] and Gorkov's theory.

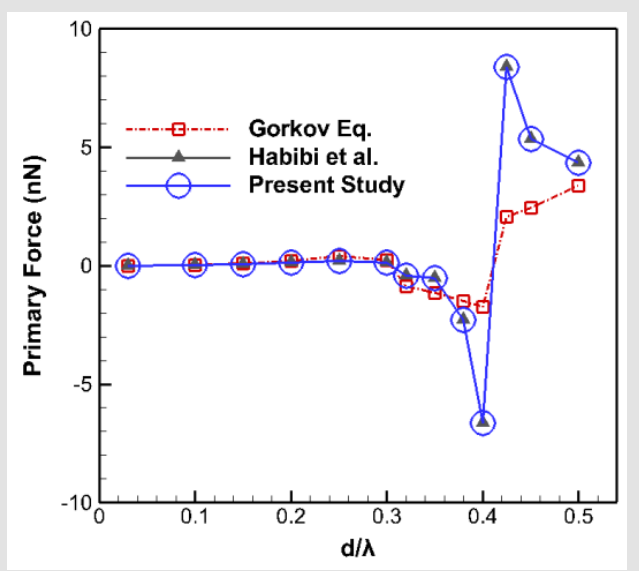

B 
Where $F_{\text {tot }}$ is the total acoustic force on each particle in the presence of two particles? $F_{\text {tot }}$ and $F_{\text {prim }}$ is the primary and the secondary acoustic forces, respectively. Accordingly, the difference between the primary and total force values resulting in the secondary acoustic force, in the current case. As shown in (Figures $5 a \& 5 b$ ) for the ratio of particle diameter to wavelength of 0.01 and 0.03 , the primary and total acoustic forces magnitude is the almost the same and indistinguishable. So, the title of y-axis in these two graphs, is primary and total force which are equivalent. Hence, the secondary acoustic force in these cases is nearly zero due to the small size and insignificant scattering effects. By increasing the particle size (Figures 5c-5e) there is an obvious difference between the primary and total acoustic force which indicates the existence of secondary acoustic force. In these cases, for small inter-particle distances (gap $<0.4$ ), secondary force is dominant and overtakes the primary force. It can be clearly seen that the present simulation follows a similar trend with good agreement. As depicted in (Figure 4a), we solve the Pressure Acoustic Physics on both the fluid and solid domains whereas [22] did not consider the solid region in the Pressure Acoustic computational domain. By doing so, comparison between the secondary acoustic force values, represents insignificant differences.

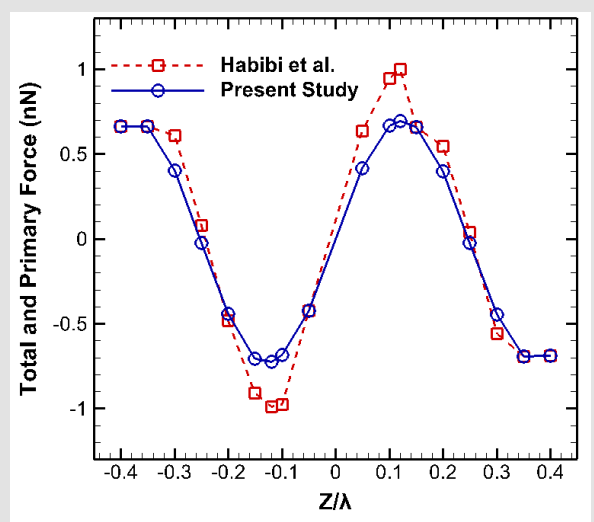

a) $\mathrm{d} / \lambda=0.01$

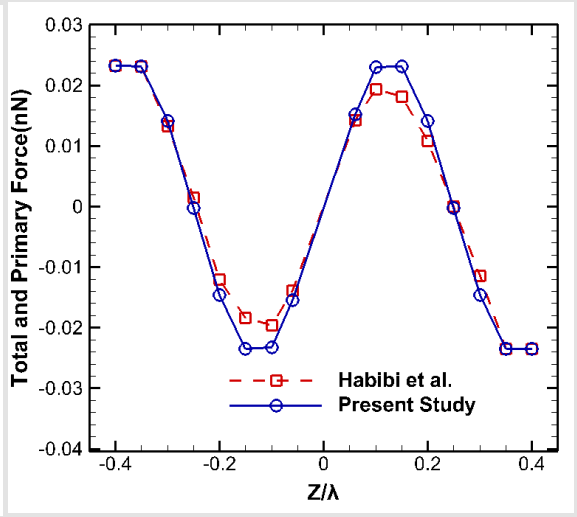

b) $d / \lambda=0.03$

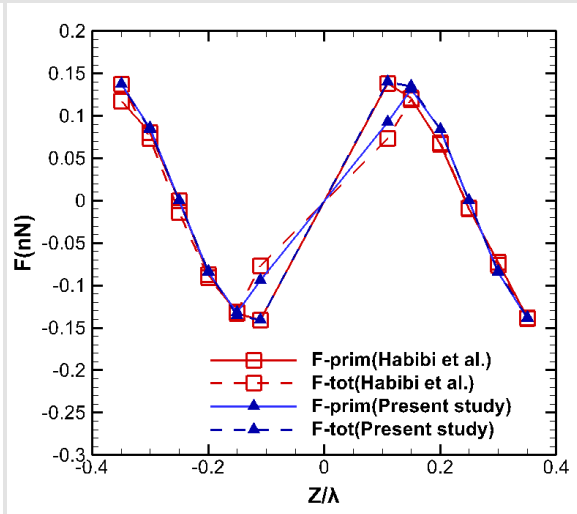

c) $\mathrm{d} / \lambda=0.2$

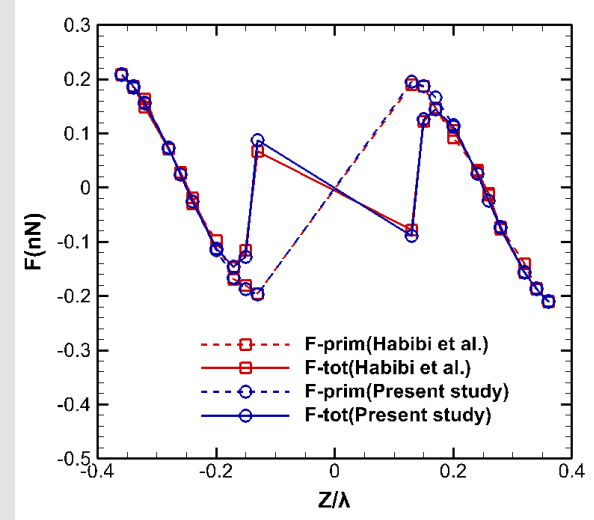

d) $\mathrm{d} / \lambda=0.25$

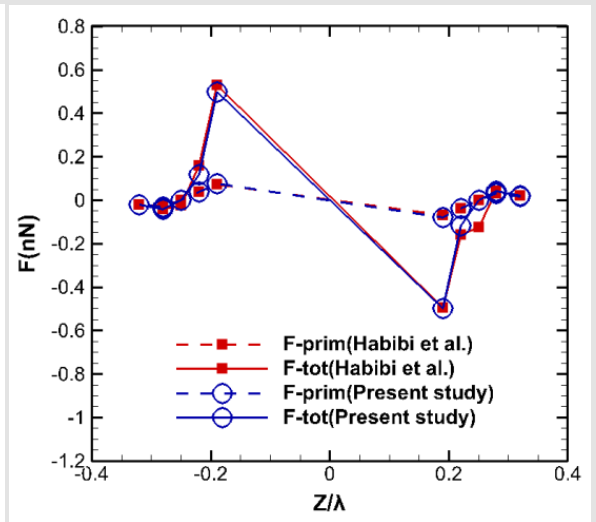

e) $\mathrm{d} / \lambda=0.3$

Figure 5: Comparison between the primary and secondary acoustic forces acting on polystyrene particles which obtained by current modeling and [22] for different ratio of particle diameter to wavelength $(d / \lambda)$ in different normalized particle positions $(\mathrm{z} / \lambda)$.

\section{Experimental Data}

We divided our results into two different sections as firstly we considered previous studies of authors [26,27] and simulated their experiments and compared their results with our evaluated data. At the second part, we make new experiments and simulate them as well and finally compare their results whit each other. The experimental procedure is exactly the same with $[26,27]$ and extensively explained in those studies. They presented a new method to measure the secondary acoustic force between particles/cells. In their method, a scattering particle is fixed in the channel and the trajectory of other particles and cells are studied in close vicinity of the fixed particle. By extracting the particle/cell trajectory and subsequently particle/cell velocity and considering Stokes drag, $F_{\text {Drag }}=6 \pi n v r_{p}$, where $n$ is dynamic viscosity, $v$ is particle velocity and $r_{p}$ is particle/cell radius, in low Reynolds number of particles, the secondary acoustic force can be calculated. 
They used the polystyrene and silica particle with diameter of 25 and $20 \mu \mathrm{m}$, respectively, as scattering points. The moving particles in close proximity of the scattering points include polystyrene with diameter of $4.8 \mu \mathrm{m}$ and RBC with diameter of $6 \mu \mathrm{m}$ for polystyrene and silica fixed particle, respectively. In that study, authors used a rectangular cross section microchannel with sizes $375 \times 110 \mu \mathrm{m}$ made of glass-silicon-glass layers.

The microchannel was excited by using the bulk acoustic wave with resonant frequency of 2-MHz. More information about the method of fixing the particle and injection the moving particle/cell into the microchannel can be found in [26,27]. In order to find the secondary acoustic force and separate that from primary acoustic force they just considered the lateral movement of moving particle in the perpendicular direction related to the wave propagation direction to calculate the Stokes drag force.

\section{Results and Discussion}

\section{Inter-Particle Force Between Polystyrene Beads}

In this section, the numerical results of interaction of 25 and $4.8 \mu \mathrm{m}$ polystyrene particles under the influence of acoustic field are compared. In order to consider the monopole and dipole effects, the polystyrene particles are located in between a pressure node and a pressure antinode. The acoustic pressure (which is generated by a plane wave and depicted in (Figure 6)and acoustic velocity are obtained by solving the 3D Helmholtz equation. As it can be seen, the acoustic pressure magnitude in the middle of channel is nearly zero which is indicated the nodal pressure plane, (Figure $6 \mathrm{a})$. The time-averaged second order pressure $(<\mathrm{P} 2>$ ) has been shown in (Figure 6b) and is approximately 1.25*105 times smaller than the magnitude of the first order pressure in (Figure 6a). This pressure acts on particles as an acoustic force and causes primary and secondary acoustic forces. The small moving and big fixed particles are shown in the (Figures 6a \& 6b). (Figure 7)shows the primary and secondary acoustic forces on moving polystyrene particle positioned at different distances from the fixed particle. The location of the moving particle in simulation are chosen exactly in the same position which extracted from the experimental data [26]. The center of the fixed particle is considered as the origin of the coordinate system as the acoustic forces act on a small particle before and after reaching the fixed particle.

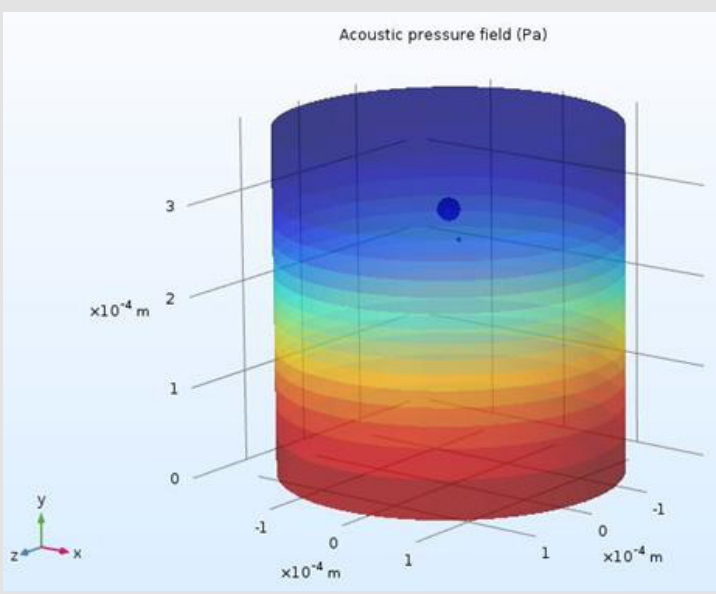

A

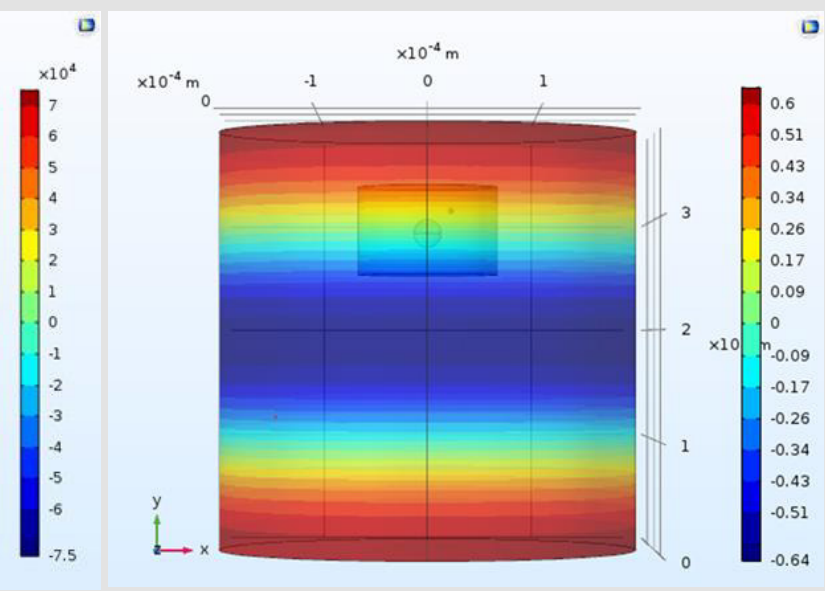

B

Figure 6: a) Color plot of acoustic pressure calculated by simulation in the 3D model at frequency of $1.95 \mathrm{MHz}$ b) Color plot of the time-averaged second-order pressure <p2> at frequency of $1.95 \mathrm{MHz}$.

It's also noticeable that when the moving particle reaches to the same level with fixed particle (position $y=0$ ), the contact angel is approximately 0 and the center-to-center line is perpendicular to the wave propagation direction. In this condition, we face with a pure secondary acoustic force acting on the small particle. As it can be seen in (Figure 7a), the primary acoustic force decreases to the minimum value when the angel $\theta$ is close to 90 . The distance between the fixed particle and the pressure nodal line is approximately $189 \mu \mathrm{m}$. This force rises again in the further locations relative to the fixed particle. (Figure $7 \mathrm{~b}$ ) illustrates that contrary to the primary acoustic force, the secondary acoustic force is significant in the vicinity of fixed particle. Actually, the trends of primary and secondary acoustic force magnitudes change in the opposite way. Comparison between the numerical and experimental results of primary and secondary forces are depicted in (Figures 7a \& 7b). The behavior of both forces from numerical results agrees with experimental data [26]. 2 Acoustic force between RBC and silica particle. In the second case, the same investigation has been done on the RBC and silica particle. The small RBC passes by the fixed silica particle. 


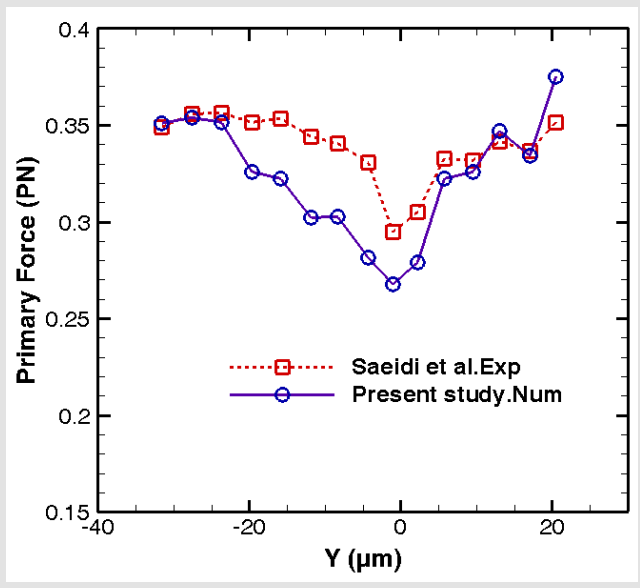

A

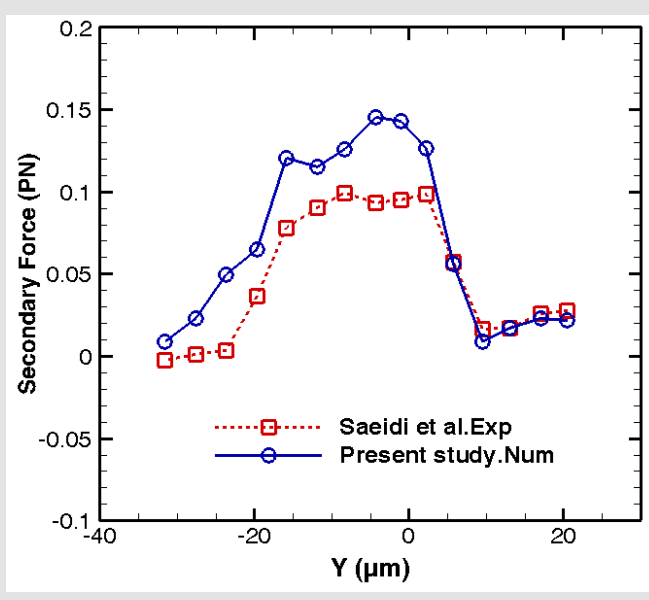

B

Figure 7: Acoustic radiation force on polystyrene particle vs. y position : blue solid line shows the numerical results and the experimental data[26] is depicted by the red dashed line a) Primary acoustic force b) Secondary acoustic force

The primary and secondary acoustic forces acting on a red blood cell are calculated and shown in (Figure 8). In this case, the silica particle with a high stiffness (E=73Gpa) shows different trend in comparison with polystyrene particle. In general, the high magnitude of Young's modulus, leads to the larger resonant frequency and in most cases, the eigenfrequencies strengthen the inter particle forces. (Figure 8b)shows that the secondary force acting on red blood cell varies from +0.1 to $-0.15 \mathrm{PN}$ while the values from polystyrene particle changes approximately between 0 and $0.1 \mathrm{PN}$. In other words, the higher stiffness of silica strongly affects the secondary acoustic force on RBC. In addition, by considering

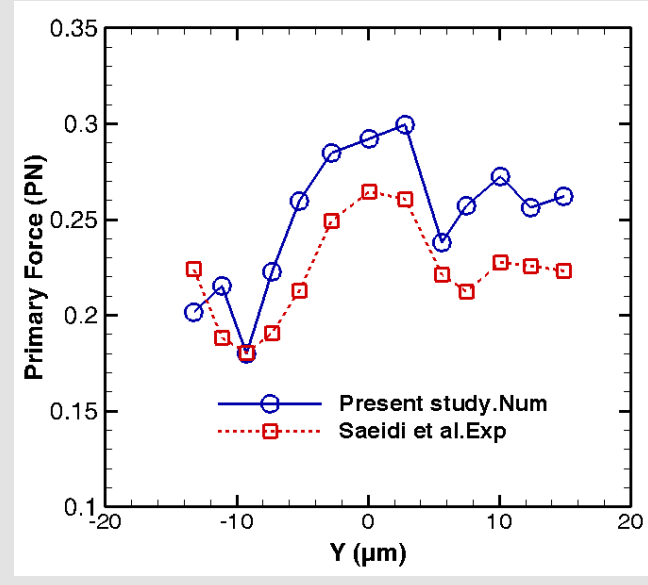

A the fact that $\mathrm{RBC}$ has weaker acoustic properties relative to the polystyrene particle, we compensate this weak property with silica particle to highlight the secondary acoustic force acting on RBC. Considering that our simulation follows the previous experimental results with good accuracy, here we implemented new experiments for more investigation. In third section, new experiments by considering the RBC and silica particle have been done and their results are summarized in (Figure 9). At all cases, (Figures 9a \& 9c) the fixed silica particle is located in the middle of the channel wall and pressure nodal line and moving RBCs are getting close to the fixed particle from the upside of the silica particle.

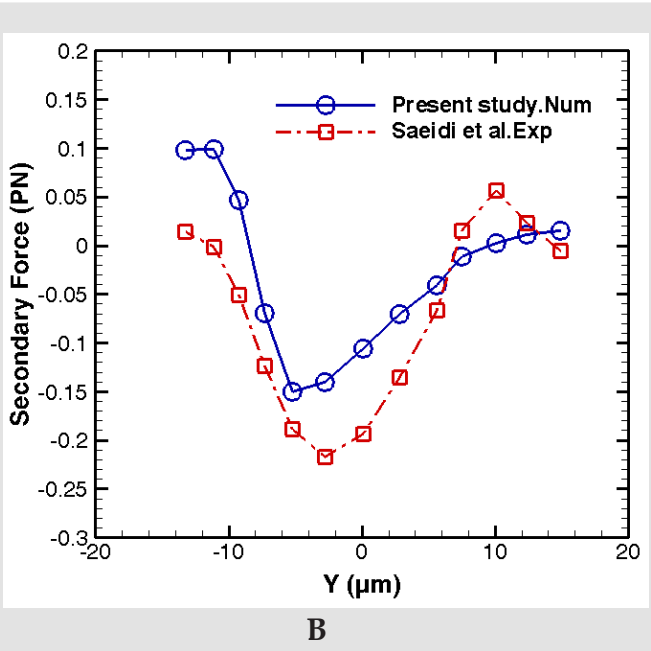

Figure 8: Acoustic radiation force on red blood cell particle vs. position and comparison with [27] a)Primary force b)Secondary force.

As it shown in all cases by decreasing the vertical distance of moving RBC to the silica particle the magnitude of the secondary acoustic force increases. In addition, in all cases almost at the vertical distances about 15-18 micrometer from fixed silica particle the magnitude of secondary acoustic force is nearly zero which indicates that the minimum effect of acoustic force in RBC trajectory. In case 1, (Figure 9a), there is a decrease in the magnitude of secondary acoustic force when the vertical distance of moving RBC is about 7 micrometers. The reason is the contact between the RBC and silica particle which reduces the RBC moving 
speed. In case $9 \mathrm{~b}$, the sudden reduction in secondary acoustic force in vertical distance of $15 \mu \mathrm{m}$ between RBC to silica particle could be related to the unexpected movement of RBC in close proximity of fixed particle based on visual observation. This unanticipated movement causes the change in center-center distance of RBC and silica particle and subsequently change in the secondary acoustic force.

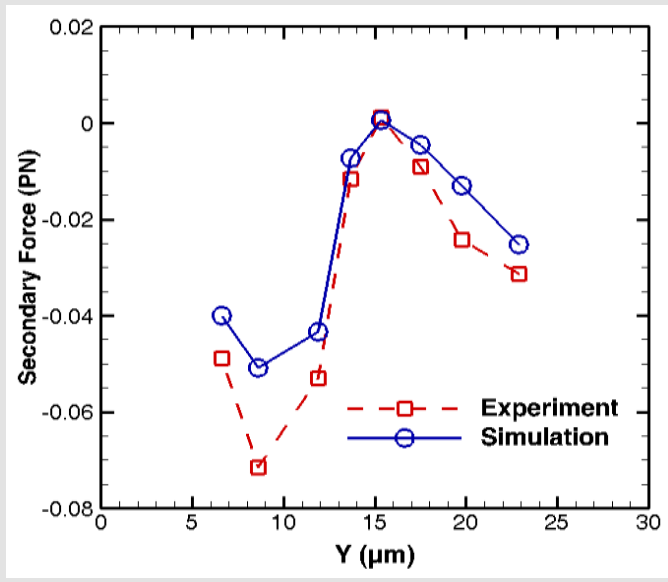

A

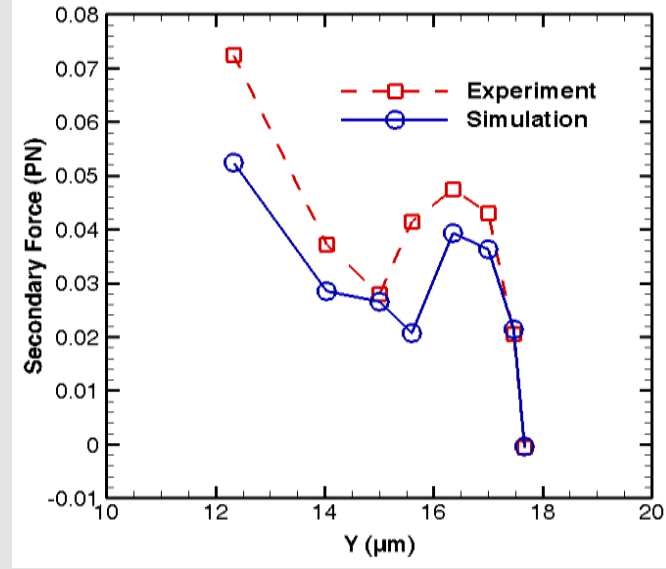

B

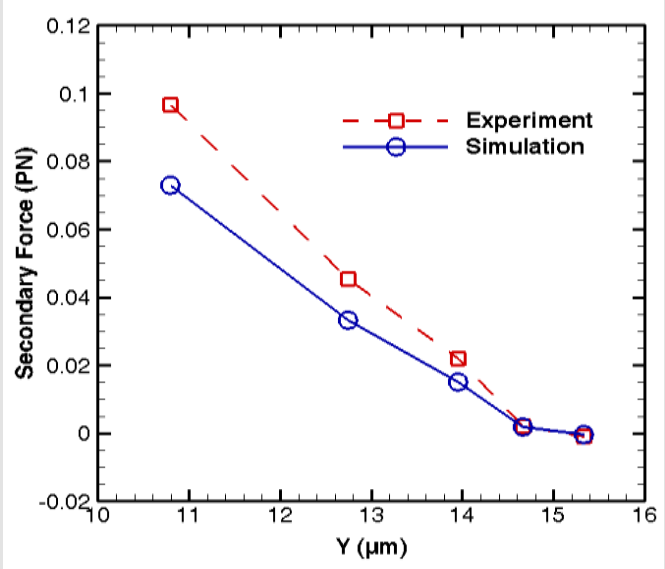

C

Figure 9: Acoustic radiation force on red blood cell particle vs. position and comparison with [27] a)Primary force b)Secondary force

\section{Conclusion}

In this paper, the secondary acoustic radiation forces acting on solid particles outside of the pressure node has been investigated both numerically and experimentally. Initially, the primary and secondary forces were determined by using a perturbation theory. We have simulated the experimental condition so as to obtain the forces acted on moving polystyrene particle. Afterward, the mentioned procedure used for silica and red blood cell. By comparison, the same behavior has been illustrated between the numerical and experimental results. The investigation of acoustic radiation force has shown that when the moving polystyrene particle is positioned close to the fixed particle, the secondary force rises significantly. It means that in the cases where the contact angel is nearly 0 and the center-to-center line is perpendicular to the wave propagation direction, the secondary force goes up, whilst the primary force decreases sharply in this region. Additionally, in the case of red blood cell and silica studied, we have observed the similar relationship between the primary and secondary forces. However, in this case, due to the larger stiffness of silica, the red blood cell has experienced the higher variation in secondary force than polystyrene particle.

\section{References}

1. Gröschl M (1998) Ultrasonic separation of suspended particles-Part I: Fundamentals. Acustica 84: 432-447.

2. Antfolk M, Laurell T (2017) Continuous flow microfluidic separation and processing of rare cells and bioparticles found in blood-A review. Anal Chim Acta 965(1): 9-35.

3. Doinikov AA (1996) On the radiation pressure on small spheres. J Acoust Soc Am 100: 1231-1233. 
4. Glynne Jones P, Mishra PP, Boltryk RJ, Hill M (2013) Efficient finite element modeling of radiation forces on elastic particles of arbitrary size and geometry. J Acoust Soc Am 133(4): 1885-1893.

5. Karlsen JT, Bruus H (2015) Forces acting on a small particle in an acoustical field in a thermoviscous fluid. Phys Rev E 92: 043010.

6. Wijaya FB, Mohapatra AR, Sepehrirahnama S, Lim KM (2016) Coupled acoustic-shell model for experimental study of cell stiffness under acoustophoresis. Microfluidics and Nanofluidics 20:69.

7. Mishra P, Hill M, Glynne Jones P (2014) Deformation of red blood cells using acoustic radiation forces. Biomicrofluidics 8(3): 034109.

8. Aghaie HR, Saghafian M, Saeidi D (2020) Elastic and Viscoelastic Modeling of Cell Deformation in Acoustically Driven Microchannel. J Applied Fluid Mech 13(6): 1901-1909.

9. Bjerknes VFK (1906) Fields of Force. (Columbia University, New York).

10. Sepehrirahnama S, Lim KM, Chau FS (2015) Numerical study of interparticle radiation force acting on rigid spheres in a standing wave. J Acoust Soc Am 137: 2614-2622.

11. Evander M, Nilsson J (2012) Acoustofluidics 20: Applications in acoustic trapping. Lab Chip 12(22): 4667-4676.

12. Mettin R, Akhatov I, Parlitz U, Ohl C, Lauterborn W (1997) Bjerknes forces between small cavitation bubbles in a strong acoustic field. Phys Rev E 56: 2924

13. Crum LA (1975) Bjerknes forces on bubbles in a stationary sound field. Acoust Soc Am 57(6): 1363-1370.

14. Doinikov AA (1999) Bjerknes forces between two bubbles in a viscous fluid. J Acoust Soc Am 106: 3305-3312.

15. Pelekasis NA, Gaki A, Doinikov A, Tsamopoulos JA (2004) Secondary Bjerknes forces between two bubbles and the phenomenon of acoustic streamers. J Fluid Mech 500: 313.

\section{ISSN: 2574-1241}

DOI: 10.26717/BJSTR.2021.37.006045

Abas Khan. Biomed J Sci \& Tech Res

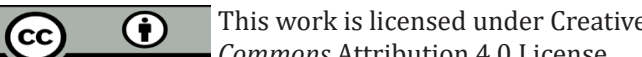

Submission Link: https://biomedres.us/submit-manuscript.php
16. Doinikov AA (2002) Viscous effects on the interaction force between two small gas bubbles in a weak acoustic field. J Acoust Soc Am 111(4): 1602-1609.

17. Zhuk A (1985) Hydrodynamic interaction of two spherical particles due to sound waves propagating perpendicularly to the center line. Sov Appl Mech 21(3): 307-312.

18. Nyborg WL (1989) Theoretical criterion for acoustic aggregation. Ultrasound Med Biol 15: 93-99.

19. Weiser M, Apfel R, Neppiras E (1984) Interparticle forces on red cells in a standing wave field. Acta Acust united Acust 56: 114-119.

20. Apfel RE (1988) Oscillations of a deformed liquid drop in an acoustic field. Am J Phys 56: 726-729.

21. Silva GT, Bruus H (2014) Acoustic interaction forces between small particles in an ideal fluid. Phys Rev E 90(6): 063007.

22. Habibi R, Devendran C, Neild A (2017) Trapping and patterning of large particles and cells in a 1D ultrasonic standing wave. Lab Chip 17: 32793290.

23. Gor'Kov L (1962) On the forces acting on a small particle in an acoustical field in an ideal fluid. In: Sov Phys Dokl (Edt.)., pp. 773-775.

24. Settnes M, Bruus H (2012) Forces acting on a small particle in an acoustical field in a viscous fluid. Phys Rev E 85: 016327.

25. Yosioka K, Kawasima Y (1955) Acoustic radiation pressure on a compressible sphere. Acta Acust united Acust 5: 167-173.

26. Saeidi D, Saghafian M, Haghjooy Javanmard S, Hammarström B, Wiklund M (2019) Acoustic dipole and monopole effects in solid particle interaction dynamics during acoustophoresis. J Acoust Soc Am 145: 3311-3319.

27. Saeidi D, Saghafian M, Haghjooy Javanmard S, Wiklund M (2020) A Quantitative Study of the Secondary Acoustic Radiation Force on Biological Cells during acoustophoresis. Micromachines 11(2): 152.

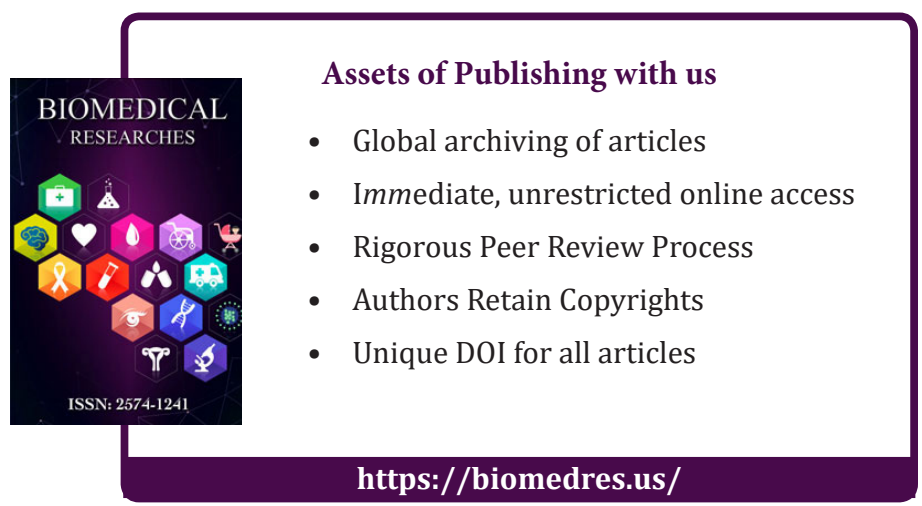

